\title{
Avaliação legal, ambiental e econômica da implantação de sistema próprio de tratamento de Resíduos de Serviços de Saúde para geração de energia em hospital-escola do Estado de São Paulo ${ }^{1}$
}

\section{Legal, Environmental and Economic Assessment to Implement a Private Biomedical Waste Treatment System to Generate Energy in Teaching Hospitals in São Paulo State}

\author{
Juliana Chiaretti NOVI* \\ Sonia Valle Walter Borges de OLIVEIRA** \\ Alexandre Pereira SALGADO JÚNIOR*** \\ Carlos Alberto Grespan BONACIM ${ }^{* * * *}$ \\ Marcio Mattos Borges de OLIVEIRA*****
}

\begin{abstract}
RESUMO
O setor hospitalar é potencial gerador dos Resíduos de Serviços de Saúde (RSS) e apresenta alto consumo de energia devido ao seu período de funcionamento e equipamentos que necessitam de infraestrutura adequada. Este artigo teve como objetivo avaliar a viabilidade legal, ambiental e econômico-financeira para implantar sistema próprio de tratamento de RSS com geração de energia em hospital de grande porte no interior do Estado de São Paulo. O hospital gera cerca de 1,9 t/dia de RSS. O tratamento por incineração com gaseificação e combustão combinadas (GCC) foi o que melhor se adequou à proposta,
\end{abstract}

\footnotetext{
" Mestra em Administração de Organizações (USP). Doutoranda do curso de Pós-Graduação em Administração de Organizações da Faculdade de Economia Administração e Contabilidade de Ribeirão Preto (FEA-RP) da Universidade de São Paulo (USP). E-mail: juliananovi@fearp.usp.br

** Doutora em Administração (USP). Professora da Faculdade de Economia Administração e Contabilidade de Ribeirão Preto (FEA-RP) da Universidade de São Paulo (USP). E-mail: soniavw@fearp.usp

*** Doutor em Administração (USP). Professor da Faculdade de Economia Administração e Contabilidade de Ribeirão Preto (FEA-RP) da Universidade de São Paulo (USP). E-mail: asalgado@usp.br

***** Doutor em Controladoria e Contabilidade (USP). Professor da Faculdade de Economia Administração e Contabilidade de Ribeirão Preto (FEA-RP) da Universidade de São Paulo (USP). E-mail: carlosbonacim@usp.br

****** Doutor em Engenharia Mecânica (USP). Professor da Faculdade de Economia Administração e Contabilidade de Ribeirão Preto (FEA-RP) da Universidade de São Paulo (USP). E-mail: mmattos@usp.br

${ }^{1} \mathrm{O}$ presente estudo teve apoio pela Fundação de Amparo à Pesquisa de São Paulo (FAPESP).
} 
com base em compilação bibliográfica, entrevistas com pesquisadores nas áreas da saúde e engenharia, órgãos técnicos e fabricantes dos equipamentos. Os resultados revelaram que a proposta está sob a égide da lei, contemplando, inclusive, a Política Nacional de Resíduos Sólidos, mas com responsabilidade contínua sobre sua utilização, a fim de se evitar danos ambientais. Para a avaliação econômico-financeira, a metodologia empregada foi a de custo-efetividade. Além do licenciamento ambiental, a proposta deve se submeter a questões políticas e à opinião pública para sua aceitação.

Palavras-chave: tratamento de Resíduos de Serviços de Saúde (RSS); geração de energia; incineração com gaseificação e combustão combinadas (GCC).

\begin{abstract}
Hospitals are large producers of Medical Waste (MW) and account for high energy consumption due to their long running hours and the running of equipment that needs adequate infrastructure. The present article aimed at the assessment of legal, environmental and economic feasibility of the implementation of a private MW treatment system to generate energy in a large hospital in the interior of São Paulo state. The hospital produces around 1.9 tons of waste per day. The treatment by means of incineration with gasification combined cycle (GCC) suited the proposal best. The research was based on compilation revision, interviews with researchers in the field of Health, Engineering, technical organizations and equipment manufacturers. Results revealed that the proposal is under the support of law and takes into consideration the National Policy for Solid Waste, but with continuous responsibility for its use so that environmental damage can be avoided. The method used for the economic-financial assessment was cost-effectiveness. Apart from environmental licensing, the proposal must be authorized by the political issues and the public opinion about the acceptance of the proposal must be taken into consideration.
\end{abstract}

Keywords: Medical Waste (MW) treatment; energy generation; incineration with Gasification Combined Cycle (GCC).

\section{Introdução}

Segundo Pesquisa Nacional de Saneamento Básico realizada em 2008 pelo Instituto Brasileiro de Geografia e Estatística (IBGE, 2010), cerca de 50,8\% dos municípios brasileiros destinavam seus resíduos sólidos urbanos (RSU) incorretamente. Em 2010, pesquisa da Associação Brasileira de Empresas de Limpeza Pública e Resíduos Especiais (ABRELPE, 2010) revelou a geração de RSU em 350 municípios, onde se constatou um aumento de $6,8 \%$ em relação a 2009 , totalizando 60.868 .080 t/ano.

A promulgação da Lei n ${ }^{\circ} 12.305$ de 2010, que instituiu a Política Nacional de Resíduos Sólidos - PNRS (BRASIL, 2010), foi importante, pois representa uma tentativa de modificar o cenário precário que acarreta efeitos adversos ao ambiente.

A atividade hospitalar é potencial geradora dos denominados Resíduos de Serviços de Saúde (RSS), que proporcionam impactos significativos ao ambiente e à saúde coletiva caso tenham uma precária e indevida disposição final, muitas vezes sem receber tratamento adequado (FERREIRA, 1995).

Além disso, devido ao porte e a atividades realizadas continuamente, alguns desses estabelecimentos possuem equipamentos que exigem uma infraestrutura adequada, o que demanda também um consumo elevado de energia.

Desse modo, novas tecnologias estão sendo desenvolvidas no intuito de se definir soluções para a problemática dos resíduos e diversificar a matriz energética (CARNEIRO, 2009). Algumas buscam obter energia proveniente dos resíduos sólidos submetidos ao processo denominado gaseificação e combustão combinadas (GCC).

Em 2009, na Polônia, foi realizado um estudo no Hospital Oncológico em Bydgoszcz para analisar o fluxo de energia gerado no processo de incineração de RSS, pois comumente esses equipamentos são utilizados para assegurar a desinfecção biológica sem, contudo, ser 
proposta uma forma de recuperação energética (BUJAK, 2009).

Entretanto, a legislação ambiental no Estado de São Paulo é rígida quanto aos sistemas de implantação para tratamento de resíduos, exigindo a abertura do processo de licenciamento ambiental junto ao órgão competente, no caso, a Companhia de Tecnologia de Saneamento Ambiental (CETESB).

Além dos riscos internos do manejo dos RSS, há também a vulnerabilidade do sistema até sua destinação final (REZENDE, 2006). No hospital pesquisado, o tipo de tratamento a que são submetidos é por micro-ondas, realizado por empresa terceirizada contratada pela Prefeitura, sendo dispostos em aterro sanitário Classe II A.

A incineração teve maior aplicação para o tratamento dos RSS. Porém, em decorrência de equipamentos obsoletos, sem manutenção ou controle de emissão de poluentes, sua aceitação gera opiniões favoráveis e desfavoráveis. O resíduo só pode ser submetido ao processo de incineração após o gerador ter realizado uma análise prévia, que consiste na sua caracterização (ABNT, 1990).

Sendo assim, ante essas questões, foi selecionado e indicado equipamento que possui a tecnologia GCC para o tratamento desses resíduos, com a possibilidade de recuperação energética a ser utilizada pelo próprio hospital.

Nesse contexto, pretende-se avaliar como podem ser tratados os RSS em sistema próprio, visando obter recuperação energética e que atenda aos aspectos legal, ambiental e econômico. Para isso, o objetivo geral foi a avaliação legal, ambiental e econômico-financeira da implantação de sistema próprio de tratamento de resíduos de serviços de saúde para geração de energia em hospital de grande porte no interior do Estado de São Paulo.

A estrutura do artigo é composta pelo referencial teórico que aborda o tema acerca da classe dos resíduos de serviços de saúde (RSS), desde as normas reguladoras e os tipos de tratamento até os aspectos relacionados às restrições legais e ao contexto econômico da avaliação econômico-financeira do estudo. A seguir, consta a metodologia do trabalho, descrevendo o tipo de pesquisa, a caracterização de seu objeto, o procedimento de coleta e análise de dados. Por fim, são apresentados os resultados e discussão, além das considerações finais.

\section{Aspectos relevantes dos Resíduos de Serviços de Saúde}

Foi realizada uma compilação da literatura existente sobre a gestão dos RSS, abordando as suas características e os principais aspectos relacionados à avaliação econômica dos tipos de tratamento disponíveis, além do pressuposto de possibilitar o desenvolvimento de um modelo de decisão mais amplo para avaliação de projetos de destinação desses RSS no setor da saúde coletiva.

\subsection{Os RSS, sua classificação e categorização}

Os Resíduos de Serviços de Saúde (RSS) constituem uma classe de resíduos inserida no grupo dos resíduos sólidos. Na PNRS de 2010, os resíduos sólidos são classificados quanto à sua origem e periculosidade. A ABNT os classifica em sua NBR 10004:2004 quanto aos seus riscos potenciais ao meio ambiente e à saúde em duas classes: Resíduos Classe I (Perigosos) e Resíduos Classe II (Não Perigosos). Esta última se subdivide em: Resíduos Classe II A (não inertes) e Resíduos Classe II B (inertes), devendo ser feita a sua caracterização (ABNT, 2004). Assim, os considerados neste estudo foram os RSS que são definidos pela ABNT na NBR 12.807:1993 (ABNT, 1993); pela RDC n 306, de 07 de dezembro de 2004, da Agência Nacional de Vigilância Sanitária-ANVISA (BRASIL, 2004); e pela Resolução $\mathrm{n}^{\circ} 358$, de 29 de abril de 2005, do Conselho Nacional do Meio Ambiente - CONAMA (CONAMA, 2005), sendo que as duas últimas Resoluções versam sobre os gerenciamentos interno e externo dos RSS, respectivamente.

A ANVISA divide-os em cinco grupos, conforme suas características ou riscos. São eles: grupo A, com riscos biológicos ou infectantes; grupo $\mathrm{B}$, com riscos químicos; grupo $\mathrm{C}$, com riscos radioativos; grupo $\mathrm{D}$, do tipo comum ou domiciliar; e grupo E, resíduos cortantes ou perfurocortantes. E para que sejam incinerados devem ser caracterizados conforme prevê a NBR 11.175:1990 da ABNT (ABNT, 1990). Segundo a Organização Pan-Americana de Saúde (OPAS) e a Organização Mundial da Saúde (OMS), o gerenciamento dos RSS engloba procedimentos a serem implantados desde a geração até 
a disposição final dos mesmos. Devem ser observadas as questões técnicas, normativas e legais, visando à redução de sua produção e à proteção dos trabalhadores que os manipulam, além da preservação da saúde pública da população, dos recursos naturais e do meio ambiente (CENTRO PAN-AMERICANO, 1997).

Li e Jenq (1993) avaliaram as características de resíduos gerados pelo National Taiwan University Hospital (NTUH) para submetê-los a tratamento por incineração. Os resultados demonstraram que os resíduos continham $38 \%$ de umidade e um poder calorífico de $3.400 \mathrm{kcal} /$ $\mathrm{kg}$, sendo que o carbono representou $34 \%$ e o oxigênio $15 \%$ dos elementos.

$\mathrm{O}$ que influencia diretamente na eficiência dos sistemas de tratamento são as características físico-químicas dos RSS, pois estão relacionadas com o poder calorífico (MATTIOLI; SILVA, 2002).

Andrade e Schalch (1997), Rego e Noda (1993) e Gil (2007) observaram em seus resultados que o poder calorífico desses resíduos variou entre $3.826 \mathrm{kcal} / \mathrm{kg}$ e $4.990 \mathrm{kcal} / \mathrm{kg}$.

Contudo, a segregação é fundamental e determinante para que a destinação final dos RSS seja adequada. Dessa forma, pode-se decidir sobre quais alternativas técnicas serão utilizadas para o tratamento desses resíduos selecionando, assim, os equipamentos que sejam mais convenientes (SCHNEIDER; ALDART; GASTALDELLO, 2000).

\subsection{Os tipos de tratamentos dos RSS utilizados no Brasil}

Eles podem ser classificados conforme técnica e tipos de aplicação, devendo visar à proteção à saúde e ao meio ambiente (CHAERUL; TANAKA, 2008). O tipo escolhido varia de acordo com o estabelecimento de serviço de saúde e com a capacidade de gerenciamento dos seus resíduos (CHENG et al., 2009).

Pesquisadores estão adotando a tomada de decisão (multivariada) como ferramenta para auxiliar na escolha do melhor sistema (KARAGIANNIDIS et al., 2010). Foram utilizados aspectos econômicos, sociais, técnicos e ambientais para decidir sobre os tipos de tratamento de
RSS que são utilizados no Brasil: incineração, autoclave e micro-ondas (DURSUN; KARSAK; KARADAYI, 2011), além da desinfecção química e tocha de plasma, que foram desconsideradas neste estudo (DIAZ; SAVAGE; EGGERTH, 2005).

A tecnologia da Gaseificação e Combustão Combinadas (GCC) pode ser aplicada a vários tipos de resíduos, transformando-os em gás combustível que pode ser utilizado para geração de energia (HCWHE, 2004).

Teoricamente, o processo de incineração, quando realizado de maneira correta, é um método de tratamento adequado para os RSS (BRIDGWATER, 1995). Contudo, devem ser considerados o potencial energético dos resíduos e as perdas de gases durante o processo de GCC: no incinerador ( $8 \%$ a $12 \%$ ) e na caldeira ( $15 \%$ a $25 \%$ ).

A Resolução CONAMA n ${ }^{\circ} 316$, de 29 de outubro de 2002 (BRASIL, 2002), dispõe sobre procedimentos e critérios para o funcionamento de sistemas de tratamento térmico de resíduos. A ABNT, em sua NBR 11.175:1990 (ABNT, 1990), também estabelece os padrões de desempenho para incineração. Para que os RSS sejam submetidos a esse processo, eles devem ser caracterizados para se conhecer os componentes tóxicos, seu poder calorífico, sua umidade, os elementos que o compõem e outros listados (ABNT, 1990).

De acordo com essa NBR, a incineração de resíduos sólidos é um processo de oxidação à alta temperatura que destrói ou reduz o volume ou recupera materiais ou substâncias e um incinerador é qualquer dispositivo, aparato, equipamento ou estrutura usado para executar esse processo.

Existem, internacionalmente, opiniões contrárias a esse tipo de tratamento. Em Portugal, deixou de ser utilizado e foram adotadas outras tecnologias para o tratamento dos RSS. Mas deve-se considerar que, com manutenção, tecnologias avançadas e práticas adequadas de manejo dos RSS, ele pode ser realizado (HCWHE, 2004).

Países como Áustria, Alemanha, Reino Unido, França e Itália fazem uso de incinerador de resíduos (MAVROPOULOS, 2010).

Em Istambul, o incinerador construído em 1995 é capaz de tratar 24 t/d de RSS, reduzindo assim quantidade a ser disposta em aterro, de acordo com Ozturk 
e Iskenderoglu² (2002, apud DURSUN; KARSAK; KARADAYI, 2011). Contudo, a grande desvantagem da incineração é a emissão de poluentes tóxicos e muitos deles causam danos às pessoas e ao meio ambiente (FICARELLA; LAFORGIA, ${ }^{3}$ 2002, apud DURSUN; KARSAK; KARADAYI, 2011).

\subsection{Restrições legais aos tipos de tratamento de RSS nos hospitais de São Paulo}

A Lei Estadual n 12.300 de 2006 (SÃO PAULO, 2006) institui a Política Estadual de Resíduos Sólidos no Estado de São Paulo. Já no âmbito nacional, foi promulgada a Lei $\mathrm{n}^{\circ} 12.305$ de 2010, que institui a PNRS (BRASIL, 2010). As normas e legislações brasileiras são exigentes com relação ao gerenciamento dos RSS, entretanto, elas acabam não sendo cumpridas na prática, principalmente pela carência de recursos do estabelecimento de saúde ou mesmo pela falta de controle e fiscalização por parte dos órgãos competentes.

Ademais, os RSS possuem classificação e definição em várias legislações e atos normativos de diversos órgãos, incluindo aquelas com relação aos tratamentos térmicos (BRASIL, 2002).

\subsection{A avaliação econômico-financeira do tipo de tratamento de resíduos sólidos: aspectos relacionados às restrições legais e ao contexto econômico}

A avaliação econômica no setor da saúde é necessária para que os recursos, cada vez mais escassos, sejam utilizados de modo eficiente. Assim, a apuração de seus custos baseia-se no conceito de custo-oportunidade. Segundo Pindyck e Rubinfeld (2006, p. 182), o custo de oportunidade são os “custos associados às oportunidades perdidas quando os recursos de uma empresa não são utilizados da melhor forma possível".
Trata-se de uma análise comparativa entre alternativas em termos de mensuração de custos e consequências dos tratamentos na área da saúde. Esta avaliação não substitui a tomada de decisão, apenas serve como um suporte que engloba técnica e valor e, também, deve ser comparada em relação a outros aspectos decisórios (DRUMMOND; STODDART; TORRANCE, 1987).

Para a realização desses exames, uma das etapas é a análise de sensibilidade, podendo ser expressa como o impacto que uma variável causa ao ser comparada a outros parâmetros que não os adotados anteriormente. Qualquer estimativa de impacto será avaliada como mais adequada quando forem determinadas as variações necessárias que poderiam modificar certa decisão (ELEUTÉRIO, 2009).

Os métodos de avaliação econômica na área da saúde buscam escolher e comparar os custos e consequências de um programa proposto, pois assim ela se torna mais completa, se baseando na escolha da alternativa que melhor se adeque aos custos de oportunidade (MELO; CENDOROGLO NETO, 2008). Por fim, a avaliação econômica dos programas de saúde deve ser aplicada em função do tipo de programa a ser desenvolvido e dos objetivos pretendidos com a execução do programa.

Contudo, conforme retratado em estudos internacionais, uma política de gestão de resíduos sólidos eficiente deve contemplar variantes normativas e tecnológicas locais (OLIVEIRA; ROSA, 2003). Nas avaliações de programas de saúde existem interferências de externalidades, algumas delas intangíveis; optou-se, na avaliação financeira, pela comparação entre o desembolso efetivo do hospital em bases mensais comparado ao gasto estimado pela decisão de operacionalizar o incinerador.

Para a análise econômica foi utilizado o método custo-efetividade, proposto por Drummond, Stoddart e Torrance (1987) em métodos para avaliação econômica dos programas de saúde. As análises de custo-eficiência dos sistemas de saúde constituem um desafio para sua realização, mas elas podem fornecer orientações sobre previsões de custos e eficácia nas áreas de tecnologia.

\footnotetext{
${ }^{2}$ OZTURK, M.; ISKENDEROGLU, A. U. Health-care waste management in Istanbul (in Turkish). ISTAC A.S., Istanbul. 2002.

${ }^{3}$ FICARELLA, A.; LAFORGIA, D. Numerical simulation of flow-field and dioxins chemistry for incineration plants and experimental investigation. Waste Management, v. 20, p. 24-49, 2002
} 
Esse tipo de avaliação está sendo utilizado para auxiliar na tomada de decisão.

A literatura sugere, com frequência, a aplicação de uma medida de eficiência relativa, que determina níveis eficientes para os mesmos recursos ou insumos (inputs), bem como para os serviços/produtos (outputs), conhecida com análise por envoltória de dados (BANKER; CHARNES; COOPER, 1984).

Essa referida análise adotaria a premissa de que os programas de saúde poderiam ser tratados como unidades comparáveis. Contudo, especificamente no caso dos projetos de tratamento dos resíduos sólidos, a variável normatização é fator crítico, já que a viabilidade dos projetos envolveria também questões legais, não apenas quesitos técnicos e/ou economicidade.

Essa característica reforça o escopo desta pesquisa, que considera as três perspectivas - legal, técnica e econômica - simultaneamente dentro do processo de tomada de decisão.

\section{Procedimentos metodológicos}

Como referência, foi considerado um hospital de grande porte situado em cidade do interior do Estado de São Paulo que presta atendimento a pacientes da cidade e região, além de outras localidades do país. O projeto foi devidamente submetido ao Comitê de Ética em Pesquisa do hospital-escola.

Sob o ponto de vista dos objetivos, este trabalho apresenta elementos de pesquisas descritivas que visam à descrição das características de determinadas populações ou fenômenos, como também à descrição de um processo em uma organização, ao estudo do nível de atendimento de entidades, levantamento de opiniões e atitudes (GIL, 2002). Foram analisados os tipos de tecnologias disponíveis no mercado para tratamento de RSS e também suas vantagens e desvantagens para a obtenção de informações técnicas e econômicas. Portanto, a partir dessas informações coletadas, foram feitos cálculos e análises do processo proposto, utilizando métodos para avaliação econômica na área da saúde.

As etapas executadas na pesquisa foram a identificação da legislação pertinente aos RSS, bem como a coleta de dados estimativos quantitativos e qualitativos dos RSS no estabelecimento de saúde estudado, avaliando a possibilidade de um sistema próprio para tratamento desses resíduos com recuperação energética.

\subsection{Caracterização do objeto da pesquisa: a unidade de análise}

O hospital onde o estudo foi desenvolvido é vinculado à Secretaria da Saúde do Estado de São Paulo e foi inaugurado com a principal função de servir como hospital-escola dentro do complexo hospitalar onde está instalado. Como tal, procura desenvolver e praticar a assistência, o ensino e a pesquisa em saúde, por meio da busca permanente da excelência, contribuindo para a melhoria da qualidade de vida da população. Zela pela assistência, pela qualidade do ensino e pelo incentivo à pesquisa.

Possui duas unidades, sendo Campus e Unidade de Emergência. Os pacientes atendidos constituem-se, basicamente, de pessoas da cidade e região, porém, comumente são atendidos pacientes de várias regiões, incluindo outros Estados e, até mesmo, de outros países, por tratar-se de um hospital de referência. Conta com 866 leitos, por isso a categoria de grande porte, uma vez que o tamanho dos hospitais geralmente é mensurado pelo número de leitos.

As especialidades médicas e variedade de serviços proporcionados à população englobam cirurgias, além de internações, exames laboratoriais, exames especializados, transfusões de sangue e refeições oferecidas. Centro de Excelência que é, possui equipamentos que exigem uma infraestrutura adequada, ou seja, demandam alto consumo de energia.

\subsection{Procedimentos de coleta de dados}

Para a coleta de dados primários, foram utilizadas entrevistas semiestruturadas com os responsáveis pelos RSS no hospital e pelo tratamento de RSS do município, além de visitas programadas com os mesmos para obtenção de dados estimados. No intuito de se obter um indicador de resíduos totais, utilizou-se a razão entre os resíduos totais (em $\mathrm{kg}$ ) e o número de dias, totalizando assim um indicador de performance. 
Algumas empresas foram contatadas, mas foram coletados dados com duas empresas fabricantes de equipamentos dos sistemas propostos com tecnologia GCC: a Empresa A, localizada na região sul do país, que possui parcerias internacionais na aplicação de tecnologias para incineração de RSS, e a Empresa B, que está localizada no interior do Estado de São Paulo. Também foram entrevistados pesquisadores e profissionais de escolas de engenharia e órgãos técnicos.

A coleta de dados secundários se baseou em busca bibliográfica e aquisição de documentos fornecidos pelo centro hospitalar pesquisado. A compilação bibliográfica foi desenvolvida com material acadêmico sobre sistemas de tratamento de RSS, processos utilizados no Brasil, tecnologias disponíveis, aspectos legais e ambientais, nacionais e internacionais relacionados ao tema de pesquisa, além de técnicas de análise de viabilidade econômica de projetos semelhantes.

\subsection{Procedimentos de análise dos dados}

Foi analisada a viabilidade em termos legais, ambientais e econômicos do tratamento dos RSS por meio da tecnologia de gaseificação e combustão combinadas (GCC) visando à produção de energia a ser utilizada no próprio hospital em estudo.

As análises quantitativas dos dados relacionados à geração de energia e avaliação do sistema de tratamento proposto foi adaptada de metodologias já desenvolvidas, com base em material bibliográfico e entrevistas com pesquisadores e técnicos da área de processos de tratamento de RSS e fabricantes dos equipamentos. Esses profissionais foram buscados em centros de pesquisa de escolas de engenharia, na CETESB, em empresas especializadas em tratamento de RSS, em fabricantes de equipamentos, entre outros.

Para a análise econômica, foi utilizado o método custo-efetividade, proposto por Drummond, Stoddart e Torrance (1987) em métodos para avaliação econômica dos programas de saúde, conforme pode ser observado na Figura 1. Segundo os autores, as análises de custo-efetividade podem fornecer uma estimativa do custo, no intuito de atingir uma meta estabelecida, sendo assim utilizadas para identificar uma tecnologia que possa, para um determinado custo, produzir o máximo de efetividade.

As demais análises, incluindo-se as qualitativas, estão relacionadas ao questionamento legal e ambiental a respeito da viabilidade de instalação do sistema, incluindo as vantagens e desvantagens dos sistemas de tratamento para RSS disponíveis, além de aspectos do gerenciamento dos resíduos. Essas análises foram realizadas por meio da técnica de análise de conteúdo.

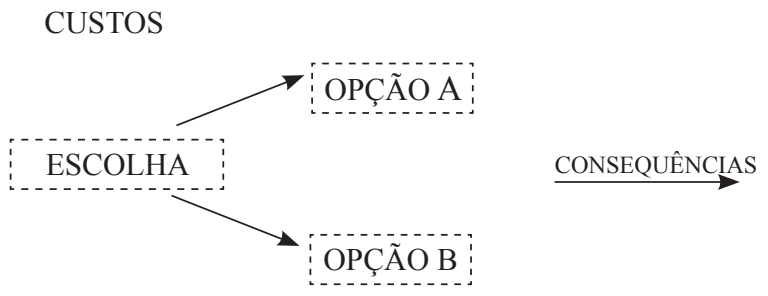

FIGURA 1 - Esquema da avaliação econômica dos Programas de Saúde.

FONTE: Elaborado pelos autores, com base em Drummond, Stoddart e Torrance (1987).

Contudo, durante a coleta de dados, restaram lacunas que foram atestadas com informações de fontes alternativas: internet, artigos técnicos ou profissionais da área. Portanto, os resultados do sistema proposto poderão ser aprimorados em estudo futuro com relação à geração de energia eficiente. Tal limitação se deve, principalmente, ao que diz respeito à caracterização dos RSS do hospital, por não ser objetivo deste artigo e, além disso, trabalhos nesse sentido são escassos.

Conforme pode ser observado, o acesso a algumas informações foi demasiado restrito. Nos órgãos públicos, informações que envolvem a gestão de resíduos eram divergentes e incompletas. Esses obstáculos não se limitaram somente à esfera pública, já que no setor privado muitas informações e dados são de propriedade dos fabricantes dos equipamentos, sendo fornecidas somente de forma estimada.

As mesmas restrições ocorrem em relação aos valores reais quanto à avaliação financeira proposta. Por se tratar de dados estimados, caso o projeto venha a ser implantado, deverão ser feitas adequações dos valores à realidade no momento de sua concretização. 


\section{Resultados e discussão}

\subsection{Os RSS no município onde está situado o hospital escola-estudado: dados estimados}

No município, a coleta dos RSS é feita por meio de uma empresa terceirizada contratada pela Prefeitura Municipal e eles são transportados até outro município, onde serão submetidos a tratamento por micro-ondas e dispostos em aterro sanitário. A coleta é feita, diariamente, nos grandes estabelecimentos geradores e, alternadamente ou quando solicitado, nos pequenos geradores, mediante o pagamento de uma taxa. Informações com relação aos valores mensais cobrados do hospital não foram fornecidas por questões éticas. Todavia, foi obtido o valor estimado com base em entrevista realizada na Secretaria do município que engloba a Divisão de Limpeza Urbana.

Os dados estimados da quantidade de RSS coletados anualmente dos estabelecimentos que solicitam o serviço na cidade e os valores pagos pelo serviço estão na Tabela 1.

Portanto, o hospital avaliado gerou cerca de 1,9 t/ dia em 2010, representando cerca de $33 \%$ do total coletado, transportado e tratado pela Prefeitura e empresa terceirizada corresponsáveis pelo processo. O sistema de tratamento realizado por micro-ondas só trata os resíduos dos Grupos A e E.
Sendo assim, o valor a ser considerado no trabalho foi o de $\mathrm{R} \$ 614 \mathrm{mil} / \mathrm{ano}$ relativo à geração de $712.080 \mathrm{~kg} /$ ano de RSS, totalizando cerca de R \$ 51.166,66 ao mês pagos pelo hospital, em estudo, à Prefeitura Municipal.

\subsection{Seleção e indicação dos tipos de tratamentos de RSS disponiveis com potencial para recuperação energética}

Inicialmente, foram observadas as alternativas inseridas nos processos térmicos disponíveis e utilizados no Brasil: autoclave, micro-ondas e incineração com o uso da tecnologia de gaseificação e combustão combinadas (GCC).

As vantagens e desvantagens dos três tipos de tratamentos estudados tiveram como referências o "Guidelines for the evaluation and assessment of the sustainable use of resources and of wastes management at Healthcare Facilities" (TOWNEND; CHEESEMAN, 2005), que demonstram as comparações entre os sistemas de tratamento de RSS (MAVROPOULOS, 2010). De acordo com os engenheiros químicos entrevistados, nem todos os resíduos classe A podem ser processados no micro-ondas, como, por exemplo, os das classes A3 e A5. Este tipo de tratamento, bem como a autoclave, aquece o resíduo e emite vapores, em geral, sem tratamento algum para a atmosfera.

TABELA 1 - DADOS ESTIMADOS DO VOLUME E DO CUSTO PARA COLETA, TRANSPORTE, TRATAMENTO E DISPOSIÇÃO FINAL DOS RSS GERADOS PELOS ESTABELECIMENTOS CADASTRADOS NO MUNICÍPIO

\begin{tabular}{lccc}
\hline & \multicolumn{3}{c}{ Valores Estimados - Estabelecimentos Cadastrados } \\
\cline { 2 - 4 } Descrição & \multicolumn{3}{c}{ Ano } \\
\cline { 2 - 4 } & 2008 & 2009 & 2010 \\
\hline & & & 6,1 \\
Média de RSS t/dia & 5,3 & 5,4 & 186,0 \\
Média de RSS t/mês & 161,5 & 165,7 & 2.232 \\
Total de RSS t/ano & 1.938 & $\mathbf{1 . 9 8 8}$ & $\mathbf{4 4 7 . 9 2 2 , 3 6}$ \\
Média mensal paga à empresa terceirizada $(\mathrm{R} \$)$ & $\mathbf{3 5 0 . 7 9 0 , 3 9}$ & $\mathbf{3 7 9 . 7 9 0 , 8 2}$ & $\mathbf{5 . 3 7 5 . 0 6 8 , 3 4}$ \\
Valor total pago à empresa terceirizada $(\mathrm{R} \$ /$ ano $)$ & $\mathbf{4 . 2 0 9 . 4 8 4 , 6 7}$ & $\mathbf{4 . 5 5 7 . 4 8 9 , 8 7}$ &
\end{tabular}

FONTE: Adaptado pelos autores, baseado em informações da Divisão de Limpeza Urbana da Prefeitura Municipal de Ribeirão Preto/SP. 
Porteous ${ }^{4}$ (2001 apud BUJAK, 2009) realizou estudos com relação às emissões gasosas a partir da incineração de resíduos e verificou que a tecnologia aplicada atualmente teve grandes avanços na questão de emissão de poluentes.

Lima e Bachmann (2002) corroboram essa assertiva. Quando há um controle de combustão eficiente somado aos sistemas de purificadores e filtros de gases, é ínfima a emissão de poluentes na atmosfera. Com isso, a opinião e a aceitação públicas tendem a ser positivas.

Segundo Teixeira (2006), pode-se obter por esse processo vantagens como redução do volume dos RSS e recuperação energética.

No Quadro 1 estão duas questões relevantes feitas aos profissionais de áreas técnicas com relação à preocupação da indicação sobre a viabilidade técnica do sistema proposto GCC.

\section{QUADRO 1-RESPOSTAS RELEVANTES DOS ENTREVISTADOS SOBRE A VIABILIDADE TÉCNICA DO SISTEMA PROPOSTO}

Pergunta: A tecnologia é adequada tecnicamente para tratar os RSS e gerar energia?

Resposta: Na realidade a gaseificação é uma etapa da incineração e por trás do nome "gaseificação" está a incineração. A gaseificação é uma tecnologia que pode ser aplicada a alguns tipos de resíduos homogêneos, assim mesmo com dificuldades, porém é inviável só para resíduos de saúde, pois eles não irão gerar gasogênio. Não há nenhum lugar em que realmente funcione a gaseificação apenas com RSS. No caso da tecnologia da Empresa A há um reator de gaseificação e combustão combinadas, ou seja, a gaseificação é a primeira etapa de um ciclo completo de incineração. A incineração sim é uma alternativa viável.

Pergunta: Qual é o potencial de geração de energia?

Resposta: Esse potencial não depende muito da tecnologia, mas sim do resíduo. Ele tem um conteúdo energético e a tecnologia tem a função de tentar aproveitá-lo da melhor maneira possível. Pode ser para gerar água quente para processos de lavagem, vapor para processos de desinfecção, ar frio para câmaras frias ou ar-condicionado e até energia elétrica. Os RSS não necessariamente possuem um alto poder calorífico e, normalmente, a quantidade também irá influenciar.

FONTE: Elaborado pelos autores com base em entrevistas realizadas com técnicos das áreas durante os meses de janeiro a agosto de 2011.
Conforme dados coletados com uma das empresas que trabalham com a tecnologia GCC, a Tabela 2 estipula os valores correspondentes à recuperação energética que se pode obter na utilização do sistema (considerando uma eficiência de combustão de $80 \%$ ).

TABELA 2 - VALORES REFERENTES À RECUPERAÇÃO ENERGÉTICA DE RESÍDUOS SUBMETIDOS AO PROCESSO COM TECNOLOGIA GCC

\begin{tabular}{cccc}
\hline $\begin{array}{c}\text { Volume de RSS } \\
\text { Tratado } \mathbf{( k g / h )}\end{array}$ & $\begin{array}{c}\text { Quantidade de água } \\
\text { quente } \mathbf{a} \mathbf{8 0}^{\mathbf{}} \mathbf{C} \mathbf{( \mathbf { l } / \mathbf { h } )}\end{array}$ & $\begin{array}{c}\text { Energia } \\
\mathbf{( k c a l / h )}\end{array}$ & $\begin{array}{c}\text { Energia } \\
(\mathbf{G L P} / \mathbf{h})\end{array}$ \\
\hline 50 & 1.800 & 108.360 & $12,3 \mathrm{~kg}$ \\
100 & 3.600 & 216.720 & $24,6 \mathrm{~kg}$ \\
150 & 5.400 & 325.080 & $36,9 \mathrm{~kg}$ \\
\hline
\end{tabular}

FONTE: Adaptado pelos autores, com base em dados fornecidos por uma das empresas que trabalham com GCC durante o mês de agosto de 2011

Um resumo sobre os tópicos abordados nessas entrevistas segue nos Quadros 2 e 3.

No processo final para a seleção do sistema de incineração com tecnologia GCC, buscaram-se as alternativas mais satisfatórias. Entretanto, cabe frisar que dependerá também de todas as implicações relacionadas às etapas de licenciamento junto aos órgãos competentes. Exames deverão ser realizados com a finalidade de caracterizar as cinzas geradas no processo proposto para posterior disposição final em aterro sanitário.

\subsection{Avaliação legal da implantação de sistema proposto com a tecnologia GCC para tratamento de RSS}

Deverá haver processo de licenciamento ambiental, pois etapas devem ser cumpridas para que os trâmites do processo conduzam à operacionalização do sistema. Elas incluem a Licença Prévia (LP), a Licença de Instalação (LI) e a Licença de Operação (LO). A Resolução SMA 079/09 (SÃO PAULO, 2009) estabelece diretrizes e condições para a operação e o licenciamento da atividade 
NOVI, J. C. et al. Avaliação legal, ambiental e econômica da implantação de sistema próprio de tratamento de Resíduos...

QUADRO 2 - TÓPICOS ABORDADOS COM RELAÇÃO AO SISTEMA PROPOSTO INCINERAÇÃO COM GCC

\begin{tabular}{|l|l|}
\hline \multicolumn{1}{|c|}{ Tópicos abordados } & Informações do incinerador com tecnologia GCC \\
\hline Custo do tratamento/investimento & Os custos são operacionais por tonelada \\
\hline Eficácia da inativação microbiana & $100,00 \%$ \\
\hline Tipos de resíduos tratados & A e B. C após decaimento e E \\
\hline Redução no volume de RSS & Até 98\% \\
\hline Uso de água & Depende do sistema de tratamento de gases e da quantidade processada \\
\hline Emissão de gases & $\begin{array}{l}\text { Os gases passam por um sistema de tratamento e as emissões são controladas por órgãos } \\
\text { ambientais. As normas para os incineradores são as mais exigentes dentre todas as normas } \\
\text { para fontes de emissão estacionárias, incluindo termoelétricas, cimenteiras e caldeiras }\end{array}$ \\
\hline Opinião pública & $\begin{array}{l}\text { A incineração tem problemas de aceitação devido à péssima qualidade dos incineradores } \\
\text { antes de haver normas e de terem sido desenvolvidas tecnologias de controle e proteção } \\
\text { ambiental. }\end{array}$ \\
\hline Fácil operação do equipamento & Com um treinamento pelo fabricante, operadores com ensino médio podem operar. \\
\hline Impacto ambiental & $\begin{array}{l}\text { Existe impacto das emissões gasosas e da disposição de cinzas em aterro. No entanto, é am- } \\
\text { bientalmente vantajoso comparado ao aterro e às tecnologias que não tratam seus gases. O } \\
\text { aterro representa um risco em longo prazo e gera emissões de metano, gás de efeito estufa. } \\
\text { As cinzas do incinerador dispostas em aterro não geram estas emissões. }\end{array}$ \\
\hline
\end{tabular}

FONTE: Elaborado pelos autores como resultado de entrevistas entre os meses de janeiro a agosto de 2011.

QUADRO 3 - TÓPICOS ABORDADOS COM RELAÇÃO AO SISTEMA UTILIZADO ATUALMENTE, MICRO-ONDAS

\begin{tabular}{|l|l|}
\hline Tópicos abordados & Dados do processo de micro-ondas \\
\hline Custo do tratamento/investimento & Segredo industrial - não revelado \\
\hline Eficácia da inativação microbiana & $99,99 \%$ \\
\hline Tipos de resíduos tratados & A e E \\
\hline Redução no volume dos RSS & Muito pouco. Ele é desumidificado \\
\hline Uso de água & Cerca de 1\% do peso dos RSS \\
\hline Emissão de gases & Vapor d'água \\
\hline Opinião pública & Muito boa, a população foi consultada. \\
\hline Fácil operação do equipamento & Sim. São 3 funcionários (3 turnos de 3 horas) \\
\hline Impacto ambiental & Pouco. RSS são triturados e dispostos em aterro Classe II \\
\hline
\end{tabular}

FONTE: Elaborado pelos autores como resultado de entrevistas entre os meses de janeiro a agosto de 2011.

de tratamento térmico de resíduos sólidos em usinas de recuperação de energia (URE). O sistema de tratamento proposto neste trabalho também está amparado por ela, pois o seu artigo $3^{\circ}$ diz que os RSS poderão ser encaminhados à URE.

Contudo, há também que se considerar questões públicas e políticas envolvidas durante a tramitação do processo.

\subsection{Avaliação econômica do sistema para tratamento de RSS com geração de energia}

Com relação aos fabricantes dos equipamentos avaliados, duas Empresas, A e B, se dispuseram a fornecer dados que contribuíssem para o trabalho.

A avaliação econômica para implantação do sistema de incineração com tecnologia GCC refere-se a 
um equipamento com capacidade nominal de $200 \mathrm{~kg} / \mathrm{h}$ de RSS da Empresa A, que utilizará querosene como combustível inicial (cerca de 3,5 litros) e enfatiza a importância de um sistema de tratamento dos gases gerados durante o processo de tratamento. Embora a Empresa B tenha contribuído significativamente para o trabalho, optou-se por seguir somente com a avaliação econômica da Empresa A, pois as informações e os valores repassados foram mais detalhados.

Assim, seguem na Tabela 3 as informações obtidas na Empresa B e, sequencialmente, o desenvolvimento das tabelas posteriores sintetiza as estimativas de custos que foram desenvolvidas a partir das metodologias de Moura, D’Ávila e Rocha Neto (1998) e da Empresa A.

TABELA 3 - CAPACIDADE NOMINAL, VALOR E CONSUMO DE ENERGIA DA EMPRESA B REFERENTES AOS EQUIPAMENTOS COM TECNOLOGIA GCC DISPONÍVEIS

\begin{tabular}{lcc}
\hline Tipo de equipamento & \multicolumn{2}{c}{ Empresa B } \\
\hline Capacidade nominal & Valor (R\$) & Consumo de energia \\
\hline $50 \mathrm{~kg} / \mathrm{h}$ & - & $7 \mathrm{kWh} / \mathrm{h}$ \\
$100 \mathrm{~kg} / \mathrm{h}$ & $625.000,00$ & $14 \mathrm{kWh} / \mathrm{h}$ \\
$150 \mathrm{~kg} / \mathrm{h}$ & $830.000,00$ & $21 \mathrm{kWh} / \mathrm{h}$ \\
\hline
\end{tabular}

FONTE: Elaborado pelos autores, com base em dados fornecidos pela Empresa B.

O montante de 1,9 t/dia de RSS gerados pelo hospital refere-se à média dos valores anuais estimados dos resíduos dos Grupos A e E que correspondem aos anos de 2008, 2009 e 2010, que são respectivamente $620 \mathrm{t}, 701 \mathrm{t}, 712 \mathrm{t}$. Os horários para funcionamento do equipamento podem ser diferenciados do avaliado neste estudo, por exemplo, de 8 horas ou 16 horas. Os parâmetros utilizados para a avaliação econômica do processo estão relacionados na Tabela 4.

Como a estimativa de benefício com a economia de energia paga pelo hospital é subjetiva, optou-se por uma comparação com valor estimado do gasto mensal do investimento em relação ao que o hospital paga atualmente pelo serviço contratado.

Informações com relação aos valores mensais cobrados do hospital não foram fornecidas. Todavia, em
TABELA 4 - PARÂMETROS UTILIZADOS NA AVALIAÇÃO ECONÔMICA COM OS VALORES ESTIMADOS PARA A IMPLANTAÇÃO DE SISTEMA PRÓPRIO DE TRATAMENTO

\begin{tabular}{lcc}
\hline Parâmetro & Índice & Empresa A \\
\hline Capacidade nominal de RSS & $\mathrm{kg} / \mathrm{h}$ & 200 \\
Regime de operação & $\mathrm{h} / \mathrm{dia}$ & 12 \\
Dias trabalhados & dias/mês & 22 \\
Quantidade de resíduos mensal & $\mathrm{t} / \mathrm{mês}$ & 52,8 \\
Investimento & $\mathrm{R} \$$ & $1.647 .550,00$ \\
Custos operacionais & $\mathrm{R} \$$ & $45.526,16$ \\
Receitas Líquidas & $\mathrm{R} \$$ & $79.200,00$ \\
Dias trabalhados por ano & dias/ano & 330 \\
Média de RSS gerados & & \\
365 dias & $\mathrm{t} / \mathrm{ano}$ & 677 \\
12 meses & $\mathrm{t} / \mathrm{mês}$ & 56 \\
30 dias & $\mathrm{t} /$ dia & 1,90 \\
\hline
\end{tabular}

FONTE: Adaptado pelos autores, com base em dados fornecidos pela Empresa A.

reportagem veiculada em janeiro de 2011 consta entrevista realizada no município com relação aos valores pagos desde os pequenos até os grandes geradores na cidade (LAPREGA, 2011). O valor citado com relação a estes últimos é de R\$614.000,00 no ano de 2010. Na Tabela 5 estão os valores para o investimento referentes à Empresa A.

As análises de custo-efetividade, com relação aos custos do hospital, identificam os processos mediante os quais os RSS se transformam em serviços para a saúde.

Com relação às obras civis, para abrigar o equipamento é necessária a construção de um galpão coberto para proteger o equipamento e que tenha uma base de concreto para suportar seu peso, sistema de energia com ligação de 380 volts trifásico e ponto de água.

Após serem identificados a quantidade e o volume dos RSS, foram elaboradas avaliações de custos fixos e variáveis para o investimento proposto. Assim, uma estimativa das implicações econômicas da implantação do sistema de tratamento por incineração com GCC pode ser analisada perante o atual sistema de micro-ondas. 
NOVI, J. C. et al. Avaliação legal, ambiental e econômica da implantação de sistema próprio de tratamento de Resíduos...

TABELA 5 - VALORES ESTIMADOS PARA O INVESTIMENTO DO INCINERADOR

\begin{tabular}{|c|c|c|c|}
\hline Investimento & Quantidade & Unidade & $200 \mathrm{~kg} / \mathrm{h}$ \\
\hline Terreno & & & 0,00 \\
\hline Taxas de Licenciamento (LP, LI, LO) & & & $30.000,00$ \\
\hline EIA/RIMA & & & $25.000,00$ \\
\hline SUBTOTAL & & RS & $55.000,00$ \\
\hline \multicolumn{4}{|l|}{ Construção civil } \\
\hline Prédio civil & $180 \mathrm{~m}^{2}$ & $\mathrm{R} \$$ & $600.000,00$ \\
\hline Bombonas & 30 & RS & $2.550,00$ \\
\hline Pavimentação, caminhos, jardinagem & & $\mathrm{R} \$$ & $130.000,00$ \\
\hline SUBTOTAL obras civis & & RS & $732.550,00$ \\
\hline \multicolumn{4}{|l|}{ Equipamentos específicos incineração } \\
\hline Sistema de incineração Luftech $200 \mathrm{~kg} / \mathrm{h}$ & & RS & $780.000,00$ \\
\hline Caldeira de recuperação & & $\mathrm{R} \$$ & $80.000,00$ \\
\hline SUBTOTAL & & RS & $860.000,00$ \\
\hline \multicolumn{4}{|l|}{ Veículos } \\
\hline Comprar & & RS & 0,00 \\
\hline Alugar & & $\mathrm{R} \$$ & 0,00 \\
\hline SUBTOTAL & & RS & 0,00 \\
\hline Total Estimado para investimento & & RS & $1.647 .550,00$ \\
\hline
\end{tabular}

FONTE: Adaptado pelos autores, baseado em informações da Empresa A.

Avaliando esses dados, verifica-se que os recursos humanos representam $28 \%$ do custo operacional. Mas o custo poderia ser reduzido para R $\$ 31.393,49$ caso fossem capacitados os funcionários do próprio hospital-escola para operar o equipamento.

Os insumos representam 21\%, mas, havendo a possibilidade de recuperação energética dos resíduos, eles também podem decair no sentido de ser utilizado vapor, água quente ou fria do processo no hospital. Os custos operacionais podem ser observados na Tabela 6 .

A manutenção representa a maior parte dos custos operacionais, totalizando 35\%. Esse valor deve receber especial atenção, uma vez que o equipamento precisa operar em excelentes condições para evitar impactos ambientais. $\mathrm{O}$ valor de depreciação corresponde a um valor econômico, devido ao seu fator subjetivo (20 anos).
Dessa forma, houve um empate técnico, sem se considerar que o investimento produz potencialmente uma receita que seria medida a partir das "economias" de energia (vapor e água quente) produzida. Houve uma tentativa de mensurar esta receita, porém, como a unidade não opera este equipamento, os resultados não foram conclusivos.

Os resultados demonstraram que o custo mensal para a decisão final de se instalar o sistema próprio para tratamento (incinerador com GCC) é de R $\$ 47.140,95$. Já o custo mensal para a decisão de se encaminhar os RSS para tratamento e disposição final solicitando esse serviço à Prefeitura foi de $\mathrm{R} \$$ 51.166,67, conforme já explicado anteriormente. Desse modo, os valores podem ser relativos, ou seja, quando comparados a outros tipos de tratamento ou comparados perante os benefícios que poderão estar implícitos na avaliação. 
TABELA 6 - CUSTOS OPERACIONAIS PARA IMPLANTAÇÃO DO SISTEMA PRÓPRIO PARA TRATAMENTO DE RSS

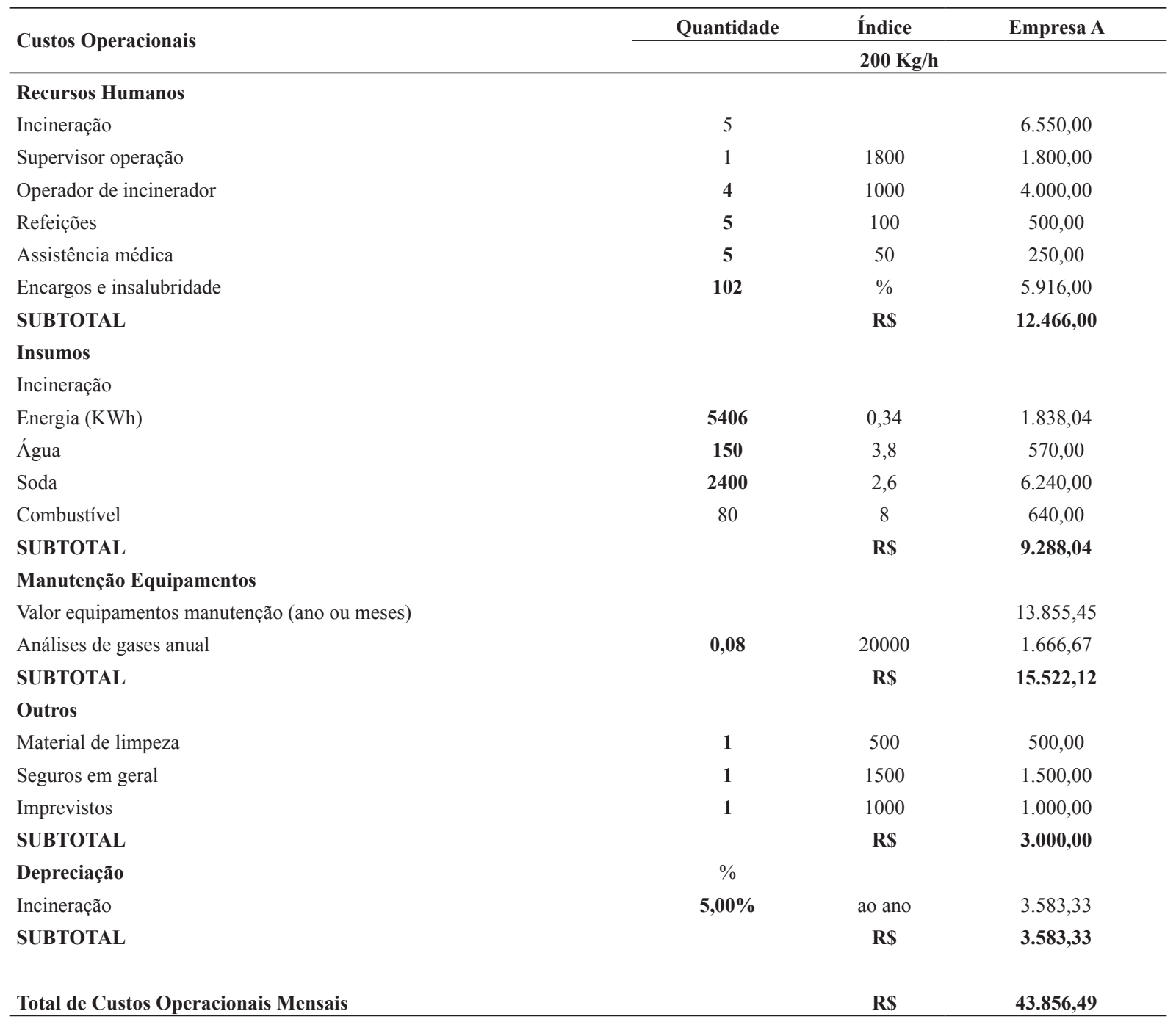

FONTE: Elaborado pelos autores, com base em informações da Empresa A.

Por fim, optou-se por avaliar o benefício implícito da decisão de implantar ou não o sistema proposto. Há indícios de que o custo para o hospital é similar ao pagamento realizado para a Prefeitura, porém não se descontaram deste custo os benefícios implícitos, tais como receita estimada de economia de energia e o con- trole operacional do equipamento, que potencialmente possibilitaria usos e destinações distintas, ou mesmo a segurança como gerador, não correndo riscos de ser corresponsável. E, ainda, os ganhos ou perdas para o meio ambiente e saúde coletiva. 


\subsection{Avaliação ambiental do sistema de incineração com tecnologia GCC}

A questão ambiental engloba também a opinião pública e as questões políticas. Com relação à opinião pública, o histórico dos incineradores no Brasil não serve de base à população. Atualmente, as questões ambientais são relevantes e a responsabilidade deve ser inerente aos envolvidos no processo.

Os incineradores utilizados até o final do século XX não possuíam a tecnologia empregada atualmente, impactando na opinião pública. Entretanto, com instalação inadequada, sem manutenção e operacionalização corretas, qualquer equipamento que realize o tratamento de resíduos poderá representar um risco.

Nos processos de micro-ondas e autoclave não há redução significativa no volume dos RSS tratados e, mesmo tendo sido esterilizados, eles poderão contaminar o solo quando dispostos em aterro sanitário, causando emissões, e isso também é um impacto a ser considerado, além de poder ser ineficaz para desinfecção de alguns micro-organismos. Contudo, para que esses impactos sejam reduzidos, inicialmente, deve haver uma correta segregação dos RSS.

Por outro lado, no processo de incineração, apesar da eliminação da patogenicidade dos RSS e da redução do seu volume, haverá liberação de gases poluentes (dioxinas e furanos) se não for devidamente controlado o processo com equipamentos apropriados. Outrossim, medidas mitigadoras podem ser implementadas para que sejam evitados possíveis impactos.

Estar em conformidade com a legislação ambiental e agir com responsabilidade é primordial para que o sistema de tratamento proposto opere corretamente.

Pode-se destacar que com relação à emissão de poluentes na atmosfera a tecnologia se mostrou avançada com relação ao controle de emissão de gases, respeitando a legislação e, principalmente, o meio ambiente. Entretanto, o equipamento necessita de manutenção adequada e pessoas capacitadas para operá-lo.

De acordo com as análises realizadas, pode-se dizer que os valores de se implantar um sistema próprio em relação ao pagamento por tratamento externo por terceiro não têm diferenças significativas. Porém, es- tudos mais minuciosos poderão ser realizados, a partir da caracterização dos resíduos do hospital, associada a um planejamento integrado em relação às possíveis formas de aproveitamento energético desse processo, bem como sobre a redução do consumo de combustíveis, emissão de gases de efeito estufa e riscos à comunidade, relacionados ao constante transporte dos resíduos por quilômetros até o local de tratamento.

Resumidamente, também como aspectos ambientais: o custo do investimento é relativo, pois depende do cenário, se composto por outros sistemas de tratamento ou se perante o meio ambiente e a sociedade; redução do risco à população, diminuindo a vulnerabilidade do sistema, eliminando o trajeto com os resíduos infectantes e perfurocortantes até o local de tratamento; redução de gases de efeito estufa pela minimização do transporte dos resíduos, que também reduz custos anuais; possibilidade de recuperação energética.

Grande parte desses aspectos também está relacionada com as avaliações feitas em relação aos aspectos legais, que não foram retomados neste item para não haver redundância.

\section{Considerações finais}

A seleção e a escolha do tipo de tratamento de RSS proposta pelo presente estudo foi a incineração com o uso da tecnologia GCC, pois, além de se tratar de um processo que poderá proporcionar recuperação energética, vez que é um processo térmico de alto aquecimento, resulta na eliminação da patogenicidade desses resíduos e na redução significativa do seu volume. Isto corrobora a ideia de reduzir tanto os acidentes provenientes da vulnerabilidade do sistema que engloba o transporte externo desses resíduos quanto a quantidade de rejeitos depositados nos aterros.

Contudo, questões relativas, por exemplo, à emissão de gases e aos riscos à comunidade também devem ser ponderadas, ainda que haja indícios de que esta tecnologia seja vantajosa.

Durante a coleta de dados, as limitações se deram principalmente no tocante à caracterização dos RSS do hospital em estudo. Nesse sentido, lacunas foram preenchidas com base em informações de fontes alter- 
nativas, tais como artigos e profissionais de áreas técnica e acadêmica.

Tecnicamente, é provável que, ao serem submetidos ao processo de incineração com GCC, os RSS poderão gerar energia em forma de vapor, água quente ou fria a ser utilizada pelo próprio hospital que, fazendo uso desse sistema de tratamento, dentro da unidade geradora, poderá reduzir os riscos que envolvem sua destinação final.

Primordial deve ser destinar os RSS com segurança, independentemente de custos ou aproveitamento energético, sendo viável sob a ótica da sociedade quanto ao aspecto ambiental. Por outro lado, caso o hospital decida por implantar o sistema próprio de tratamento de RSS, pode obter vantagens, tais como, por exemplo, além da recuperação energética, maior controle sobre a geração dos seus RSS, não sofrer sanção penal caso ocorra algum tipo de acidente durante a destinação final resultante de erro de terceiros, sendo viável sob a ótica do empreendedor.

Nos processos de micro-ondas e autoclave não há redução significativa no volume dos RSS tratados e, mesmo tendo sido esterilizados, eles poderão contaminar o solo quando dispostos em aterro sanitário, causando emissões e isso também é um impacto a ser considerado, além de poder ser ineficaz para desinfecção de alguns micro-organismos. Contudo, para que esses impactos sejam reduzidos, inicialmente, deve haver uma correta segregação dos RSS.

Por outro lado, no processo de incineração, apesar da eliminação da patogenicidade dos RSS e da redução do seu volume, haverá liberação de gases poluentes

\section{Referências}

ANDRADE, J. B. L.; SCHALCH, V. Características física e química dos Resíduos de Serviços de Saúde gerados em um estabelecimento hospitalar de grande porte. In: CONGRESSO BRASILEIRO DE ENGENHARIA SANITÁRIA E AMBIENTAL, 19., Foz do Iguaçu, set. 1997. Anais..., p. 1843-1854. Disponível em: <http://www.bvsde.paho.org/bvsacd/abes97/ sersaude.pdf $>$. Acesso em: 10/05/2011.

ASSOCIAÇÃO BRASILEIRA DE EMPRESAS DE LIMPEZA PÚBLICA E RESÍDUOS ESPECIAIS - ABRELPE. Pano- (dioxinas e furanos) se não for devidamente controlado o processo com equipamentos apropriados. Outrossim, medidas mitigadoras podem ser implementadas para que sejam evitados possíveis impactos.

Legalmente, por considerar a recuperação energética dos resíduos, também é contemplada a PNRS, todavia, a aceitação ou não da instalação do equipamento no complexo hospitalar também engloba questões políticas e opinião pública.

Sob o aspecto econômico, o investimento demonstrou ser viável, mesmo sem se considerar os ganhos com a recuperação energética proveniente dos RSS ou benefícios intangíveis. $\mathrm{O}$ investimento foi avaliado a partir de uma análise conservadora, apoiada em metodologias validadas. A questão dos custos de oportunidade de um investimento desse porte é relativa, pois, perante o custo de outros tipos de tratamento, talvez ele seja mais oneroso para o estabelecimento hospitalar; todavia, perante a sociedade e o meio ambiente, ele pode ser vantajoso, por conter benefícios implícitos que se pautam na intangibilidade.

Sendo assim, esse aspecto gera um benefício implícito que é a decisão de se implantar ou não o sistema proposto, pois aspectos intangíveis são imensuráveis. Têm-se vantagens e desvantagens nas escolhas.

Com efeito, este presente estudo não esgota a avaliação ambiental. O modelo de decisão proposto pode ser aprimorado com o desenvolvimento de estudos que incorporem variáveis representativas das condições técnicas, demográficas, padrão de consumo e de renda da região atendida pelo hospital.

rama dos resíduos sólidos no Brasil - 2010. 2010. Disponível em: $<$ http://www.abrelpe.org.br/downloads/Panorama2010.pdf >. Acesso em: 18/08/2011.

ASSOCIAÇÃO BRASILEIRA DE NORMAS TÉCNICAS - ABNT. NBR - 11.175: Incineração de resíduos sólidos perigosos - padrões de desempenho. Rio de Janeiro: ABNT, 1990.

. NBR - 12.807: Coleta de Resíduos de Serviços de Saúde. Rio de Janeiro: ABNT, 1993. 
NOVI, J. C. et al. Avaliação legal, ambiental e econômica da implantação de sistema próprio de tratamento de Resíduos...

. NBR - 10.004: Resíduos sólidos - Classificação. Rio de Janeiro: ABNT, 2004.

BANKER, R. D.; CHARNES, A., COOPER, W. W. Some models for estimating technical and scale inefficiencies in Data Envelopment Analysis. Management Science, v. 9, n. 30, p. 126-135, 1984.

BRASIL. Ministério do Meio Ambiente. Conselho Nacional do Meio Ambiente-CONAMA. Resolução n. 316, de 29.10.2002. Dispõe sobre procedimentos e critérios para o funcionamento de sistemas de tratamento térmico de resíduos. Disponível em: $<$ http://www.mma.gov.br/port/CONAMA/res/res02/res31602. html>. Acesso em: 15/03/2009.

. Ministério da Saúde. Agência Nacional de Vigilância Sanitária-ANVISA. RDC n. 306, de 07.12.2004. Dispõe sobre o regulamento Técnico para o Gerenciamento de Resíduos de Serviços de Saúde. Disponível em: < http://www.ANVISA.gov. br/e-legis/>. Acesso em: 15/03/2009.

. Ministério do Meio Ambiente. Conselho Nacional do Meio Ambiente-CONAMA. Resolução n. 358, de 29.04.2005. Dispõe sobre o tratamento e a disposição final dos resíduos de serviços de saúde e dá outras providências. Disponível em: $<$ http://www.mma.gov.br/port/CONAMA/res/res05/res35805. pdf $>$. Acesso em: 15/03/2009.

Lei n. 12.305, de 02 de agosto de 2010. Institui a Política Nacional de Resíduos Sólidos e dá outras providências. Disponível em: <http://www.planalto.gov.br/ccivil_03/_ ato2007-2010/2010/lei/112305.htm>. Acesso em: 25/08/2010.

BRIDGWATER, A. V. The Technical and Economic Feasibility of Biomass Gasification for Power Generation. Fuel, v. 74, n. 5, p. 631-653, 1995.

BUJAK, J. Experimental study of the energy efficiency of an incinerator for medical waste. Applied Energy, v. 86, n. 11, p. 2386-2393, nov. 2009.

CARNEIRO, P. A. Análise das tecnologias para gestão e reaproveitamento energético dos resíduos urbanos para reciclagem de plásticos. 2009. 112 f. Dissertação (Mestrado em Engenharia de Energia) - Universidade Federal de Itajubá, Itajubá, MG, 2009.

CENTRO PAN-AMERICANO DE ENGENHARIA SANITÁRIA E CIÊNCIAS DO AMBIENTE. Guia para o manejo interno de resíduos sólidos em estabelecimentos de saúde. Tradução de Carol Castillo Argüello. Brasília, DF: Organização Pan-Americana da Saúde - OPAS, 1997. Disponível em: <http://www.opas.org.br/sistema/arquivos/reshospi.pdf $>$. Acesso em: fev. 2011.
CHAERUL, M.; TANAKA, M. A system dynamics approach for hospital waste management. Waste Management, v. 28, n. 2, p. 442-449, 2008.

CHENG, Y. W.; SUNG, F. C.; YANG, Y.; LO, Y.H.; CHUNG, Y. T.; LI, K.-C. Medical waste production at hospitals and associated factors. Waste Management, v. 29, n. 1, p. 440-444, jan. 2009.

DIAZ, L. F.; SAVAGE, G. M.; EGGERTH, L. L. Alternatives for the treatment and disposal of healthcare wastes in developing countries. Waste Management, v. 25, p. 626-637, 2005.

DRUMMOND, M. F.; STODDART G. L.; TORRANCE, G. W. Methods for the economic evaluation of health care programmes. New York: Oxford University Press, 1987.

DURSUN, M.; KARSAK, E. E.; KARADAYI, M. A. A fuzzy multi-criteria group decision making framework for evaluating health-care waste disposal alternatives. Expert Systems with Applications, v. 38, n. 9, p. 11453-11462, Sep. 2011.

ELEUTÉRIO, J. P. L. Proposta de um modelo de negócio para a implantação de um sistema para tratamento de Resíduos de Serviço de Saúde. 2009. 118 f. Dissertação (Mestrado em Engenharia de Produção) - Universidade Estadual Paulista UNESP, Bauru, 2009.

FERREIRA, J. A. Resíduos sólidos e lixo hospitalar: uma discussão ética. Cadernos de Saúde Pública, v. 11, n. 2, p. 314-320, 1995.

GIL, A. C. Como elaborar projetos de pesquisa. 4. ed. São Paulo: Atlas, 2002.

GIL, T. N. L. Caracterização qualitativa e quantitativa dos resíduos de serviços de saúde gerados na Irmandade Santa Casa de Misericórdia de São Carlos, SP. 2007.85 f. Graduação (Engenharia Ambiental) - Universidade de São Paulo - USP, São Carlos, 2007.

HEALTH CARE WITHOUT HARM EUROPE - HCWHE. Non-Incineration Medical Waste Treatment Technologies in Europe. 2004. Disponível em: <http://www.noharm.org/details. cfm?type $=$ document $\& I D=1183>$. Acesso em: 27/11/2010.

INSTITUTO BRASILEIRO DE GEOGRAFIA E ESTATÍSTICA - IBGE. Ministério do Planejamento, Orçamento e Gestão. Pesquisa Nacional de Saneamento Básico 2008. Rio de Janeiro, 2010. Disponível em: <http:/www.ibge.gov.br/home/ estatistica/populacao/condicaodevida/pnsb2008/PNSB_2008. pdf>. Acesso em: 25/08/2010.

KARAGIANNIDIS, A.; PAPAGEORGIOU, A.; PERKOULIDIS, G.; SANIDA, G.; SAMARAS, P. A multi-criteria 
assessment of scenarios on thermal processing of infectious hospital wastes: A case study for Central Macedonia. Waste Management, v. 30, n. 2, p. 251-262, Feb. 2010.

LAPREGA, M. R. Lixo hospitalar gera R 27 milhões em dívidas - Acúmulo de 7 anos, débito com a prefeitura envolve hospitais públicos, filantrópicos e particulares da cidade. Jornal Folha de São Paulo. 11 de janeiro de 2011; Folha Ribeirão: C1.

LI, C. S.; JENQ, F. T. Physical and chemical composition of hospital waste. Infection Control and Hospital Epidemiology, v. 14, n. 3, p. 145-150, 1993.

LIMA, R.; BACHMMAN, R. T. Pollutant emissions from modern incinerators. International Journal of Environment and Pollution, v. 18, n. 4, p. 336-345, 2002.

MATTIOLI, C. E.; SILVA, C. L. Avaliação de parâmetros na implantação de processos para tratamento de Resíduos Sólidos de Serviços de Saúde. In: SIMPÓSIO ÍTALO-BRASILEIRO DE ENGENHARIA SANITÁRIA E AMBIENTAL, 6., Vitória, ES. Anais... 10 p., set. 2002.

MAVROPOULOS, A. Estudo para gestão de Resíduos de Serviços de Saúde no Brasil. Relatório Final. Epem SA. Set. 2010. 71 p. Disponível em: <http://www.abrelpe.org.br/arquivos/estudo_gestao.pdf $>$. Acesso em: 19/08/2011.

MELO, J. C.; CENDOROGLO NETO, M. Avaliação de Tecnologias da Saúde (ATS) e suas implicações na prática clínica. Einstein: Educação Contínua em Saúde, v. 6, n. 3, pt. 2, p. 132-134, 2008.

MOURA, J. C.; D’ÁVILA, S. G.; ROCHA NETO, T. Processo de gaseificação e combustão combinadas (GCC) para disposição final de resíduos industriais. In: CONGRESSO BRASILEIRO DE ENGENHARIA QUÍMICA, 12., Porto Alegre, RS, 1998. Anais... Porto Alegre: COBEQ, 1998. Disponível em: <http://www.termoquip.com.br/>. Acesso em: 10/06/2011.

OLIVEIRA, L. B.; ROSA, L. P. Brazilian waste potential: energy, environmental, social and economic benefits. Energy Policy, v. 31, n. 14, p. 1481-1491, 2003.
PINDYCK, R. S.; RUBINFELD, D. L. Microeconomia. 6. ed. São Paulo: Prentice Hall, 2006.

REGO, R. C. E.; NODA, R. Caracterização preliminar de resíduos sólidos de estabelecimentos hospitalares. In: SEMINÁRIO INTERNACIONAL SOBRE RESÍDUOS SÓLIDOS HOSPITALARES, 12. Cascavel-PR, Anais..., p. 6-22, nov. 1993.

REZENDE, L. R. Vulnerabilidade dos geradores de resíduos de saúde frente às Resoluções 358 CONAMA e RDC 306 ANVISA. O Mundo da Saúde, v. 30, n. 4, p. 588-597, out./dez. 2006.

SÃO PAULO. Lei Estadual n. 12.300, de 16.03.2006. Institui a Política Estadual de Resíduos Sólidos e define princípios e diretrizes. Disponível em: < http://www.al.sp.gov.br/portal/geral/ddilei/DdiLeiListaDetalhe.jsp?idLgLei=61778\&textoBusca=> . Acesso em: 17/03/2009.

Secretaria de Estado do Meio Ambiente. Gabinete do Secretário e Assessorias. Resolução SMA 079, de 04.11.2009. Estabelece diretrizes e condições para a operação e o licenciamento da atividade de tratamento térmico de resíduos sólidos em Usinas de Recuperação de Energia - URE. Diário Oficial do Estado de São Paulo. São Paulo, 2009.

SCHNEIDER, V. E.; ALDART, V.; GASTALDELLO, M. E. T. A Caracterização de Resíduos de Serviços de Saúde como ferramenta para o monitoramento de sistemas de gestão destes resíduos em estabelecimentos hospitalares. In: CONGRESO INTERAMERICANO DE INGENIERIA SANITÁRIA Y AMBIENTAL, 27., Porto Alegre, 3-8 dez 2000. Anais... Porto Alegre, RS, p. 1-7, 2000.

TEIXEIRA, G. P. A gestão dos Resíduos de Serviços de Saúde no município de Juiz de Fora, MG. GABES/MA. Seção Maranhão da ABES. In: SEMINÁRIO NACIONAL DE RESÍDUOS SÓLIDOS. Responsabilidade socioambiental. Tema III - Resíduos Sólidos - Gerenciamento/Manejo, 8 p., São Luiz, MA, 2006. Anais... São Luiz: ABES, 2006.

TOWNEND, C. R.; CHEESEMAN, C. R. Waste Management \& Research, v. 23, p. 398-408, 2005.

Recebido em 20 de agosto de 2012. Aceito em 20 de fevereiro de 2013. Publicado em junho de 2013. 